\section{On the Approximation of Delayed Systems by Taylor Series Expansion}

\section{Tamas Insperger}

Department of Applied Mechanics,

Budapest University of Technology and Economics,

Budapest, Hungary

e-mail: insperger@mm.bme.hu

It is known that stability properties of delay-differential equations are not preserved by Taylor series expansion of the delayed term. Still, this technique is often used to approximate delayed systems by ordinary differential equations in different engineering and biological applications. In this brief, it is demonstrated through some simple second-order scalar systems that low-order Taylor series expansion of the delayed term approximates the asymptotic behavior of the original delayed system only for certain parameter regions, while for high-order expansions, the approximate system is unstable independently of the system parameters.

[DOI: $10.1115 / 1.4027180]$

Keywords: time delay, Taylor series, asymptotic behavior, stability

\section{Introduction}

Time-delay systems often arise in different engineering applications. A feedback mechanism typically involves time delays; cars following traffic models [1], delayed networks [2,3], crane payload stabilization [4], or flutter instability [5] can be mentioned as practical examples. Time delays are also often used to model mechanical contact problems such as wheel shimmy [6] or regenerative machine tool chatter [7]. Analysis of qualitative properties of delay-differential equations (DDEs) is therefore a highly important task in engineering $[8,9]$.

DDEs of the form

$$
\dot{x}(t)=f(t, x(t), x(t-\tau)), \quad x \in \mathbb{R}^{m}
$$

are often approximated by ordinary differential equations (ODEs) through Taylor series expansion of the delayed term in powers of $\tau$. Although such an approximation might provide satisfactory results for certain parameter combinations (typically for small delays), it cannot be justified by rigorous mathematical theorems. The Taylor series expansion of the delayed term about $\tau=0$ reads

$$
\begin{aligned}
x(t-\tau)= & x(t)-\tau \dot{x}(t)+\frac{1}{2} \tau^{2} \ddot{x}(t) \mp \ldots+(-1)^{n} \frac{1}{n !} \tau^{n} x^{(n)}(t) \\
& +R(t, \tau, n)
\end{aligned}
$$

where the remainder term is

$$
R(t, \tau, n)=(-1)^{n+1} \frac{1}{(n+1) !} \tau^{n+1} x^{(n+1)}(t-\theta \tau)
$$

with $\theta \in[0,1]$. An $n$ th-order approximation is obtained by neglecting the remainder term $R(t, \tau, n)$. Such an approximation, however, has no mathematical foundations at all. The remainder term is typically not small, and the asymptotic behavior of the original DDE is usually completely different from that of the ap-

Contributed by the Design Engineering Division of ASME for publication in the Journal of Computational and Nonlinear Dynamics. Manuscript received December 31, 2013; final manuscript received March 12, 2014; published online January 12, 2015. Assoc. Editor: Parviz Nikravesh. proximate ODE [10-12]. Furthermore, this approximation involves higher-order derivatives, which may not even exist for the original delayed system.

If Eqs. (2) and (3) are substituted directly into Eq. (1) without neglecting the remainder term, then one gets a differential equation of advanced type since the highest derivative (i.e., $x^{(n+1)}$ ) appears with a delayed argument. These equations are known to have infinitely many characteristic exponents on the right half of the complex plane. In this sense, equations of advanced type are always strongly unstable [10]. This feature also reflects the inability of the Taylor series expansion to approximate time-delayed terms.

Still, there are several articles in the literature that apply Taylor series approximations for the delayed terms. In some special cases, this approximation can actually be justified. If the delay $\tau$ is sufficiently small compared to the characteristic time of the system then replacing the term $x(t-\tau)$ by $x(t)$ (zeroth-order expansion) or by $x(t)-\tau \dot{x}(t)$ (first-order expansion) provides a good approximation $[13,14]$. The first-order approximation is also often used for stochastic time-delay systems to eliminate the delay from the equation [15]. If the coefficient of the delayed term is small [16], then the Taylor series approximation can also be valid for large delays as well. In human balancing models with reflex delay, the delayed terms are often approximated by either first-order $[17,18]$ or second-order [19,20] Taylor series expansion. In these cases, however, the delay is not necessarily small compared to the characteristic time of the system, and the approximation may lead to qualitatively different behavior.

The goal of this technical brief is to demonstrate that Taylor series expansion of the delayed term may lead to a qualitatively different system. Two case studies are presented. First, a series of stability diagrams are presented for a second-order system with delayed proportional feedback in order to show the differences between the asymptotic behavior of the approximations of different order for different delays. Second, the critical delay, which limits the stabilizability of an unstable second-order system by delayed proportional-derivative (PD) control, is analyzed for approximations of different order.

\section{The Delayed Oscillator}

Consider the equation

$$
\ddot{x}(t)+a x(t)=p x(t-\tau)
$$

where $a$ is the system parameter, $p$ is the proportional gain, and $\tau$ is the delay. This system is often referred to as a delayed oscillator and its stability properties are often represented by stability charts plotted in the plane $(\tau, p)[4,5,21]$. Note that, without restriction of generality, one can assume that $a=1$. Taylor series expansion of the delayed term up to the $n$ th-order gives the ODE

$$
\begin{aligned}
\ddot{x}(t)+a x(t)= & p x(t)-p \tau \dot{x}(t)+\frac{1}{2} p \tau^{2} \ddot{x}(t)-\frac{1}{6} p \tau^{3} x^{(3)}(t) \\
& \pm \ldots+(-1)^{n} \frac{1}{n !} \tau^{n} x^{(n)}(t)
\end{aligned}
$$

As it can be seen, in the case of higher than second-order Taylor series expansion, the approximation introduces new leading derivatives. These new terms show up with alternating sign, consequently, expansion of high-order will result in an unstable ODE, which can easily be seen by means of the Routh-Hurwitz criteria. Stability analysis of the original DDE (4) and its approximate ODE (5) can be performed using standard tools, e.g., by Dsubdivision method, and the number of unstable characteristic exponents can also be determined [21]. The corresponding stability charts indicating the asymptotic behavior of the different expansions can be seen in Fig. 1. As it is shown, the zeroth-order expansion (i.e., when the time delay is totally neglected) approximates the asymptotic behavior of the original DDE only at certain 

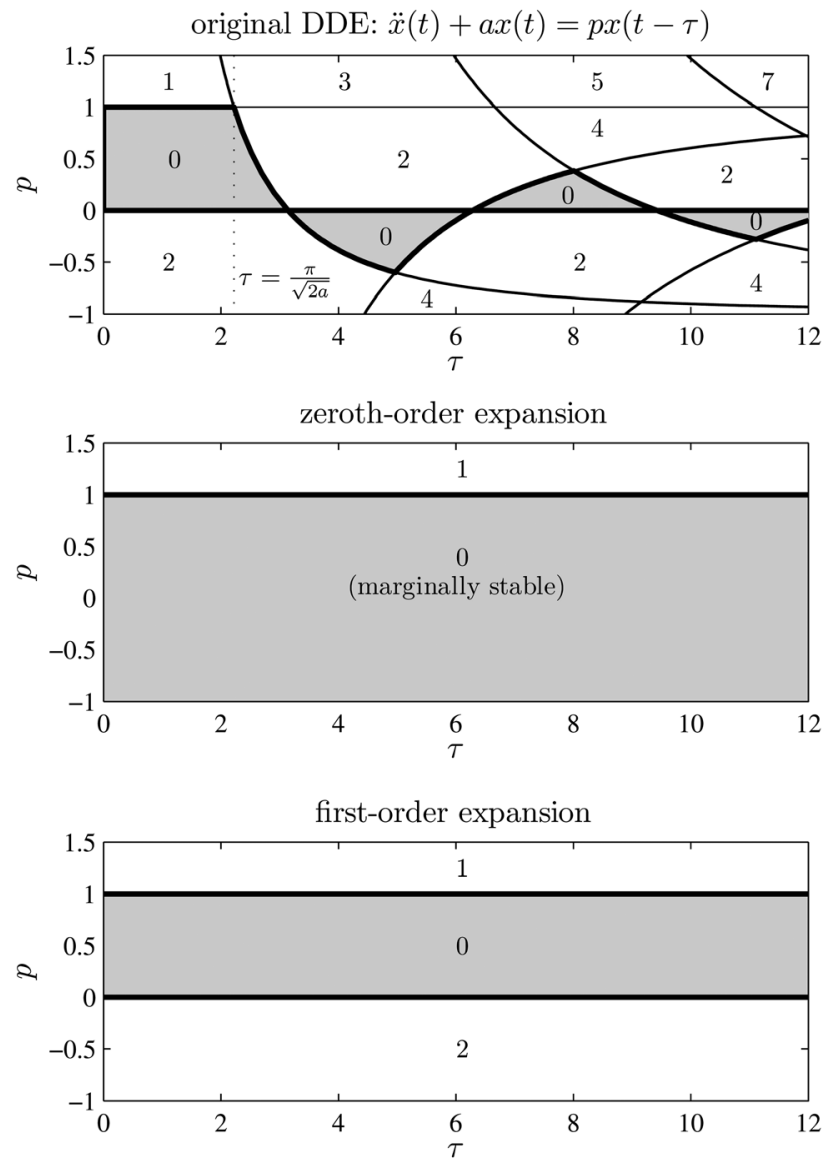
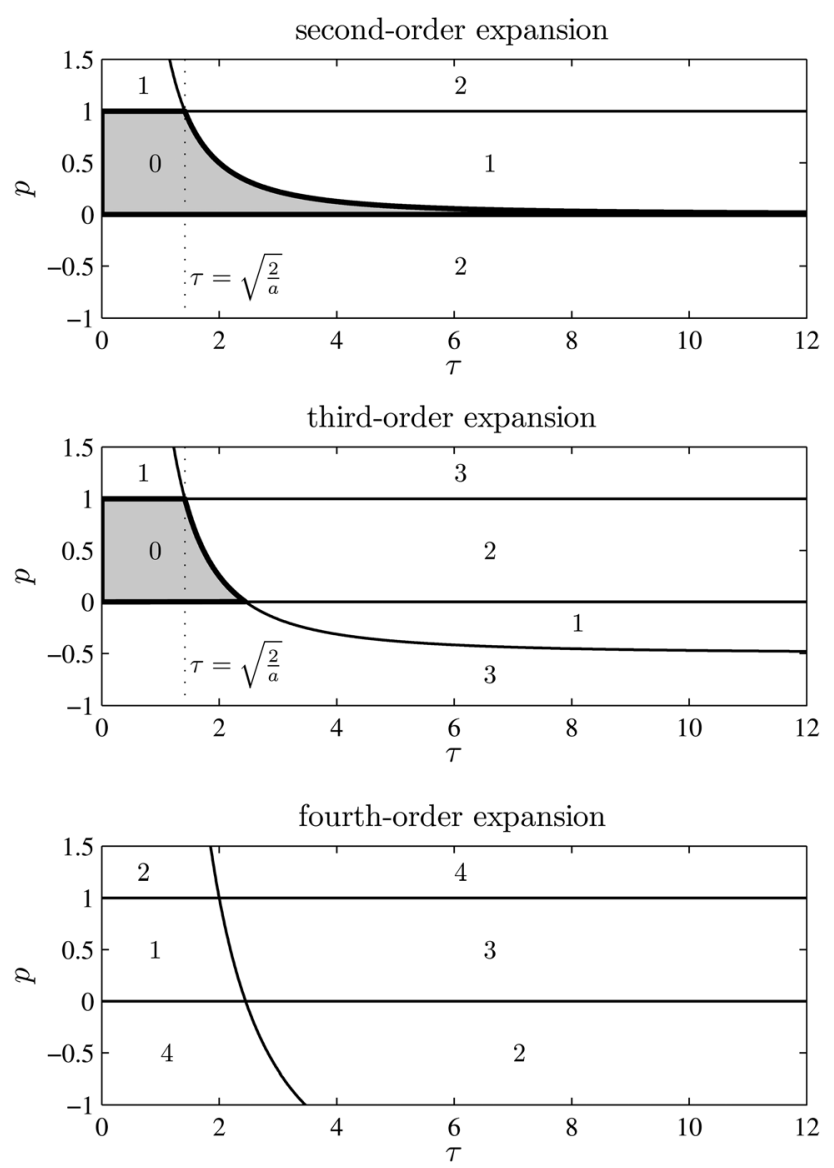

Fig. 1 Stability diagram and the number of unstable characteristic exponents for the delayed oscillator (4) with $a=1$ and its Taylor series approximations of different order. Stable domains are indicated by gray shading.

parameters regions, e.g., if $\tau<\pi / \sqrt{2 a}$ and $p>0$. The first-, the second-, and the third-order expansions provide a better approximation for small delays. The first-order expansion provides an exact asymptotic behavior for any proportional gain $p$ if $\tau<\pi / \sqrt{2 a}$ ). Similarly, the asymptotic behavior of the secondand third-order expansions matches that of the original one if $\tau<\sqrt{2 / a}$. For large delay values, however, the asymptotic behavior is completely different. Fourth- and higher-order expansions yield an unstable system independently of the delay and the gain parameters. The divergence in the stability properties for Taylor series approximation can also be seen by the number of unstable characteristic exponents. The higher the order of the approximation, the larger the number of unstable characteristic exponents for different parameter regions.

\section{Stabilizability by Delayed PD Control}

Consider now the equation

$$
\ddot{x}(t)-a x(t)=-p x(t-\tau)-d \dot{x}(t-\tau)
$$

where $a>0$ is the system parameter, $p$ is the proportional gain, $d$ is the derivative gain, and $\tau$ is the feedback delay. This equation describes a delayed PD control of an unstable second-order plant. It is known that there is a critical delay $\tau_{\text {crit }}=\sqrt{2 / a}$, which limits stabilizability: if the feedback delay is larger than $\tau_{\text {crit }}$, then the system is unstable for any combinations of $p$ and $d[8,21]$. Note that similarly to the delayed oscillator, one can assume that $a=1$ without restriction of generality. Taylor series expansion of the delayed terms up to the $n$ th-order gives the ODE

$$
\begin{aligned}
\ddot{x}(t)-a x(t)= & -p\left(x(t)-\tau \dot{x}(t)+\frac{1}{2} \tau^{2} \ddot{x}(t) \mp \ldots+(-1)^{n} \frac{1}{n !} \tau^{n} x^{(n)}(t)\right) \\
& -d\left(\dot{x}(t)-\tau \ddot{x}(t)+\frac{1}{2} \tau^{2} x^{(3)}(t)\right. \\
& \left.\mp \ldots+(-1)^{n} \frac{1}{n !} \tau^{n} x^{(n+1)}(t)\right)
\end{aligned}
$$

Conditions for stability and stabilizability and the number of unstable characteristic exponents can be determined by the analysis of the corresponding characteristic functions. The stability diagrams for the original DDE (6) and its approximate ODE (7) are shown in Fig. 2 for $a=1$ and $\tau=0.5$. The number of unstable characteristic roots is also presented. For the first- and the secondorder expansions, the stability diagrams show some similarities to that of the original DDE, however, for higher than the third-order approximations, no stable parameter regions are obtained. Actually, the higher the order of the approximation, the larger the minimum number of the unstable characteristic exponents.

The critical time delay can be determined for each case by some straightforward calculations. If the time delay is increased, then the stability boundaries are moving and at the critical delay $\tau=\tau_{\text {crit }}$, the stable domain typically shrinks to a single point. The critical time delays for the different approximations are shown in Fig. 2. In the case of the zeroth-order expansion, the critical delay is infinity since in this case the delay is simply neglected. In the first-order expansion, which is often used for the problem of delayed PD control $[17,18]$, the critical delay is smaller by a factor of $1 / \sqrt{2}$ than the one corresponding to the original DDE. The second-order expansion actually gives the exact critical delay 

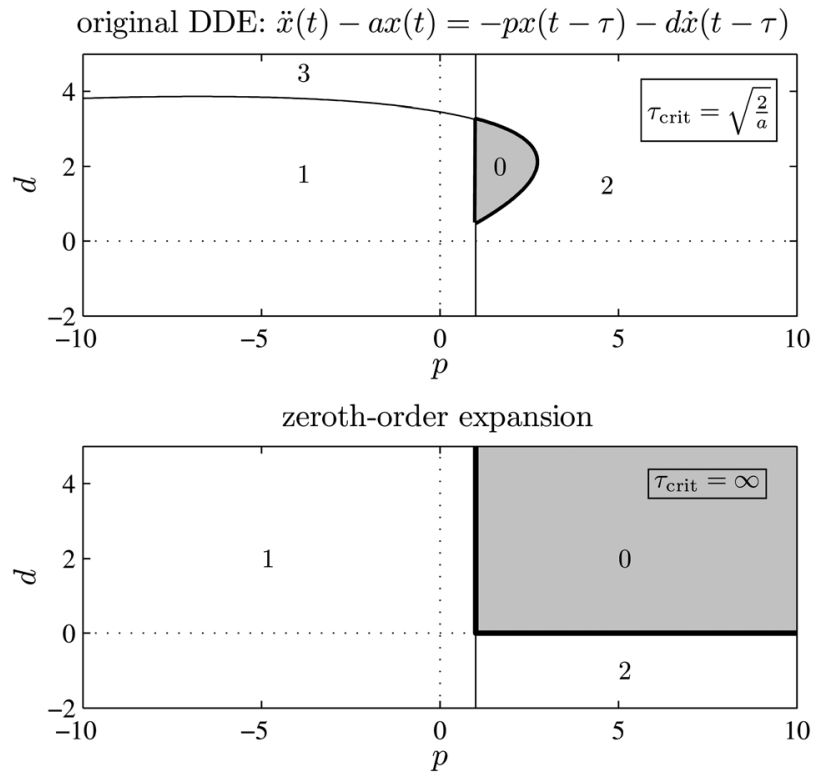

first-order expansion

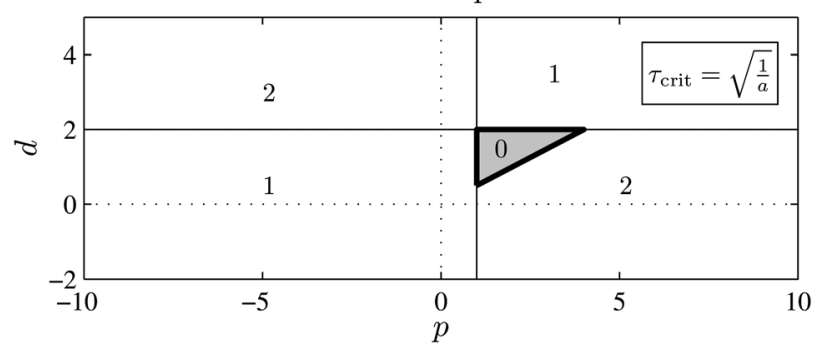

second-order expansion

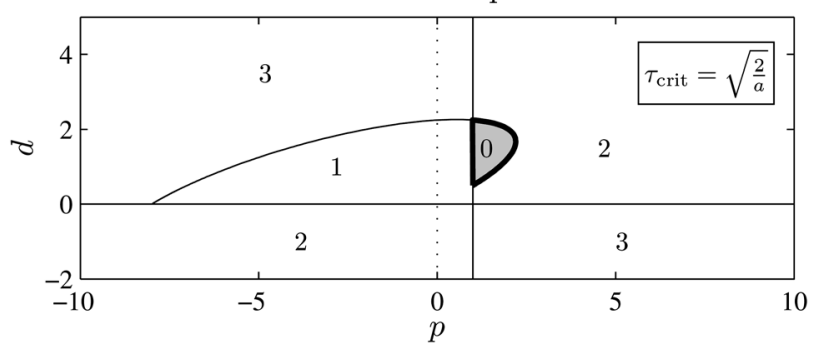

third-order expansion

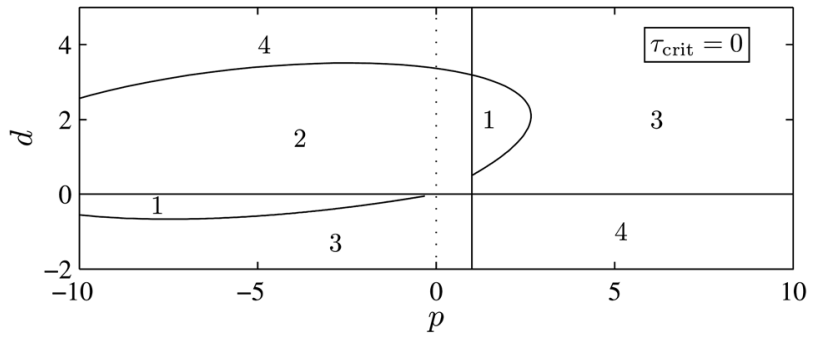

fourth-order expansion

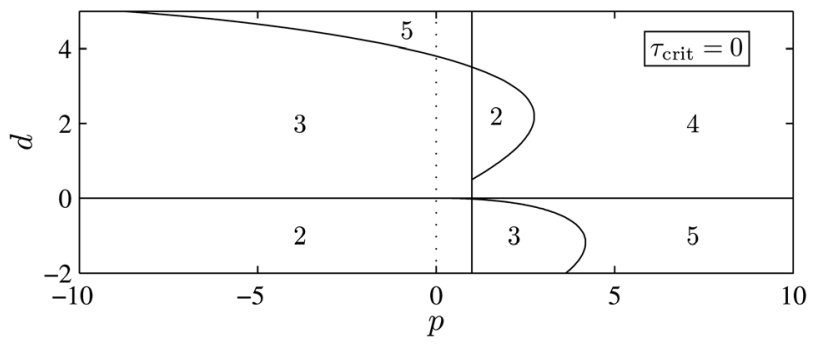

Fig. 2 Stability diagram and the number of unstable characteristic exponents for Eq. (6) with $a=1$ and $\tau=0.5$ and its Taylor series approximations of different order. Stable domains are indicated by gray shading. The critical delay for the different approximations is also shown.

$\tau_{\text {crit }}=\sqrt{2 / a}$. The critical delay for the third- and higher-order expansion is zero since in this case the approximate system is unstable for any pair $(p, d)$. This analysis demonstrates that, similarly to stability properties, stabilizability conditions are not preserved either by higher-order Taylor series approximations.

\section{Conclusions}

Although Taylor series expansion of the delayed term is often used in different applications, it is not a justified technique to approximate delayed systems $[10,12]$. In this technical brief, this fact was demonstrated using two case studies: the delayed oscillator and the delayed PD control of an unstable second-order system. Although first- and second-order Taylor series expansions of the delayed term may approximate the asymptotic behavior of DDEs for certain parameter regions, the accuracy of the approximation is radically reduced by higher-order expansions. If the order of the Taylor series expansion exceeds the order of the leading derivative by 2 , then the approximate system becomes unstable independently of the system parameters. Taylor series approximation of the delayed term is therefore not always an appropriate tool to analyze asymptotic behavior of time-delay systems and should be applied to particular problems with special attention.

\section{Acknowledgment}

This work was supported by the Hungarian National Science Foundation under Grant No. OTKA K105433.

\section{References}

[1] Orosz, G., Wilson, R. E., and Stepan, G., 2010, "Traffic Jams: Dynamics and Control," Philos. Trans. R. Soc., A, 368(1928), pp. 4455-4479.

[2] Mao, X., and Hu, H., 2010, "Stability and Bifurcation Analysis of a Network of Four Neurons With Time Delays," ASME J. Comput. Nonlinear Dynam., 5(4), p. 041001 .

[3] Szalai, R., and Orosz, G., 2013, "Decomposing the Dynamics of Heterogeneous Delayed Networks With Applications to Connected Vehicle Systems," Phys. Rev. E, 88(4), p. 040902.

[4] Erneux, T., and Kalmar-Nagy, T., 2007, "Nonlinear Stability of a Delayed Feedback Controlled Container Crane," J. Vib. Control, 13(5), pp. 603-616.

[5] Ma, H., and Butcher, E. A., 2005, "Stability of Elastic Columns With Periodic Retarded Follower Forces," J. Sound Vib., 286, pp. 849-867.

[6] Takacs, D., and Stepan, G., 2009, "Experiments on Quasiperiodic Wheel Shimmy," ASME J. Comput. Nonlinear Dynam., 4(3), p. 031007.

[7] Bachrathy, D., Turi, J., and Stepan, G., 2011, "State Dependent Regenerative Effect in Milling Processes," ASME J. Comput. Nonlinear Dynam., 6, p. 041002.

[8] Insperger, T., and Stepan, G., 2011, Semi-Discretization for Time-Delay Systems, Springer, New York.

[9] Bobrenkov, O. A., Nazari, M., and Butcher, E. A., 2012, "Response and Stability Analysis of Periodic Delayed Systems With Discontinuous Distributed Delay," ASME J. Comput. Nonlinear Dynam., 7(3), p. 031010.

[10] Èl'sgol'c, L., 1964, Qualitative Methods in Mathematical Analysis, American Mathematical Society, Providence, RI.

[11] Mazanov, A., and Tognetti, K. P., 1974, "Taylor Series Expansion of Delay Differential Equations-A Warning,” J. Theor. Biol., 46(1), pp. 271-282.

[12] Driver, R., 1977, Ordinary and Delay Differential Equations, Springer-Verlag, New York.

[13] Kurzweil, J., 1971, "Small Delays Don't Matter," Proceedings of the Symposium on Differential Equations and Dynamical Systems, Lecture Notes in Mathematics, D. Chillingworth, ed., Springer, pp. 47-49.

[14] Driver, R. D., Sasser, D. W., and Slater, M. L., 1973, "The Equation $x^{\prime}(t)=a x(t)+b x(t-\tau)$ With "Small” Delay," Am. Math. Mon., 80(9), pp. 990-995. 
[15] Guillouzic, S., L'Heureux, I., and Longtin, A., 1999, "Small Delay Approximation of Stochastic Delay Differential Equations," Phys. Rev. E, 59(4), pp. 3970-3982.

[16] Morrison, T. M., and Rand, R. H., 2007, "2:1 Resonance in the Delayed Nonlinear Mathieu Equation,” Nonlinear Dyn., 50(1-2), pp. 342-352.

[17] Stepan, G., and Kollar, L., 2000, "Balancing With Reflex Delay," Math. Comput. Model., 31(4-5), pp. 199-205.

[18] Asai, Y., Tasaka, Y., Nomura, K., Nomura, T., Casidio, M., and Morasso, P., 2009, "A Model of Postural Control in Quiet Standing: Robust Compensation of Delay-Induced Instability Using Intermittent Activation of Feedback Control," PLoS ONE, 4, e6169.

[19] Qu, X., Nussbaum, M. A., and Madigan, M. L., 2007, “A Balance Control Model of Quiet Upright Stance Based on an Optimal Control Strategy," J. Biomech., 40(16), pp. 3590-3597.

[20] Paoletti, P., and Mahadevan, L., 2012, "Balancing on Tightropes and Slacklines," J. R. Soc. Interface, 9(74), pp. 2097-2108.

[21] Stepan, G., 1989, Retarded Dynamical Systems, Longman Scientific \& Technical and John Wiley \& Sons, NY. 